\title{
Dishevelled proteins and CYLD reciprocally regulate each other in CML cell lines
}

\author{
Ceyda Çalışkan $^{1,2} \cdot$ Melek Pehlivan $^{1,3} \cdot$ Zeynep Yüce $^{1} \cdot$ Ogun Sercan $^{1}$
}

Received: 15 January 2017 / Accepted: 19 August 2017 / Published online: 24 August 2017

(c) Springer Science+Business Media B.V. 2017

\begin{abstract}
Dishevelled (Dvl) proteins are activated by Wnt pathway stimulation and have crucial roles in the regulation of $\beta$-catenin destruction complex. CYLD is a tumor suppressor and a deubiquitination enzyme. CYLD negatively regulates the $\mathrm{Wnt} / \beta$-catenin signaling pathway by deubiquitinating Dvl proteins. Loss of function and mutations of CYLD were linked to different types of solid tumors. Loss of function in CYLD is associated with Dvl hyper ubiquitination, resulting in the transmission of Wnt signaling to downstream effectors. $\beta$-catenin upregulation is observed during disease progression in chronic myeloid leukemia (CML). Deregulated Dvl signaling may be a reason for $\beta$-catenin activation in CML; and CYLD may contribute to Dvl deregulation. First, we evaluated mRNA expression in three CML cell lines and mRNA expression of the CYLD gene was found to be present in all (K562, MEG01, KU812). Unlike solid tumors sequencing revealed no mutations in the coding sequences of the CYLD gene. DVL genes were silenced by using a pool of siRNA oligonucleotides and gene expression differences in CYLD was determined by RT-PCR and western blot. CYLD protein expression decreased after Dvl silencing. An opposite approach of overexpressing Dvl proteins resulted in upregulated CYLD expression. While previous reports have described CYLD as a regulator of
\end{abstract}

Ogun Sercan

ogun.sercan@deu.edu.tr

1 Department of Medical Biology and Genetics, Faculty of Medicine, Dokuz Eylul University, Balcova, 35340 Izmir, Turkey

2 Present Address: Department of Molecular Biology, Izmir Institute of Technology, Balcova, 35340 Izmir, Turkey

3 Present Address: Vocational School of Health Sciences, Izmir Katip Celebi University, Izmir, Turkey
DVL proteins; our data suggests the presence of a more complicated reciprocal regulatory mechanism in CML cell lines.

Keywords Wnt/ $\beta$-catenin signaling pathway $\cdot \mathrm{CML}$. CYLD - Dishevelled

\section{Introduction}

The $\beta$-catenin/Wnt signaling pathway is critically involved in embryogenesis, development, cell proliferation, differentiation, self-renewal and homeostasis. Pathway activation leads to $\beta$-catenin being stabilized in the cytoplasm and translocated to the nucleus where it regulates the activity of several transcription factors [1]. This stabilization is provided through an important scaffold protein family called Dishevelled proteins (Dvls). In mammals there are three members of the Dvl family: Dvl1, -2 and -3. These proteins act as a scaffold where other proteins bind and transmit Wnt signals to downstream effectors of the Wnt signaling pathway $[2,3]$. Dvl proteins function in both canonical and non-canonical Wnt pathways. They are activated by phosphorylation in response to Wnt signaling; whereas the ubiquitination of Dvl proteins leads to an effective inhibition of the $\beta$-catenin destruction complex [4]. It has been postulated that over/ deregulated Dvl expression may play a role in the progression of several cancers [5-8]. As a result it demonstrates a potential target for cancer therapy.

The CYLD (lysine 63 deubiquitinase/ubiquitin carboxylterminal hydrolase/cylindromatosis) gene product is a deubiquitinating enzyme. It was first described as a mutated gene involved in cylindromatosis and has been classified as a tumor suppressor [9]. CYLD is identified as a negative regulator of several signaling pathways including the NF-KB pathway [10] and Dvl mediated signaling [11]. 
CYLD itself is constitutively active and its deubiquitinase domain has specificity for Lys63 chains. CYLD binds to Dvl and mediates its ubiquitination. While ubiquitination of Dvl proteins results in $\beta$-catenin accumulation (by inhibiting the $\beta$-catenin destruction complex), de-ubiquitination by CYLD will lower $\beta$-catenin levels in the cytoplasm. Several reports have shown that silencing/depleting CYLD leads to $\beta$-catenin accumulation in the cytoplasm, Wnt $/ \beta$-catenin signaling activation and the hyper ubiquitination and overexpression of Dvl proteins [11]. Genetic aberrations and reduced expression of CYLD has been reported in several solid tumors $[12,13]$. Sequence changes can occur by frame shift, nonsense, missense and splice-site mutations [14]. Recent studies reveal that CYLD deficiency may promote the generation of different solid tumors. Interestingly we have little knowledge on CYLD expression and function in hematological malignancies.

The first description of deregulated Wnt signaling in a hematological malignancy was reported in chronic myeloid leukemia (CML) [15]. CML is a clonal hematopoietic stem cell (HSC) disorder, characterized by unregulated growth of myeloid lineage cells. The hallmark of the disease is the reciprocal translocation between the long arms of chromosomes 9 and 22 that results in a BCR-ABL chimeric gene [16]. The product of this gene is a constitutively active tyrosine kinase which provides a survival signal for the cell [17]. Studies have reported activated Wnt signaling in all stages of $\mathrm{CML}$ and demonstrated that activation of the pathway plays an important role in disease pathogenesis. A previously uninvestigated underlying mechanism of $\beta$-catenin hyper activation would be the possibility deregulation of Dvl proteins through CYLD. In this study we aimed to investigate CYLD gene mutations in CML cell lines and to explore the interaction between Dvl and CYLD proteins.

\section{Materials and methods}

\section{Cell culture}

BCR/ABL positive cell lines K562, MEG01 and KU812 were purchased from DSMZ (Deutsche Sammlung von Mikroorganismen und Zellkulturen $\mathrm{GmbH}$ ). Cell lines were grown in RPMI-1640 medium containing 10\% fetal bovine serum, $2 \mathrm{mM}$ L-glutamine, $1 \mu \mathrm{g} / \mathrm{ml}$ penicillin. All cultures were incubated at $37{ }^{\circ} \mathrm{C}$ in a humidified incubator with $5 \%$ $\mathrm{CO}_{2}$. Cells were counted by Cell Scapter2.0 (Millipore, Billerica, MA).

\section{RNA extraction, RT-PCR and real time PCR}

Total RNA extractions were done by using the RNeasy Plus Mini Kit (Qiagen, Hilden), according to the manufacturer's
Table 1 Primer sequences for mRNA expression of CYLD and $\beta$-actin

\begin{tabular}{ll}
\hline Primer sequences & $\begin{array}{l}\text { Length of } \\
\text { PCR product } \\
(\mathrm{bp})\end{array}$ \\
\hline$\beta$-actin & 318 \\
F: ATCATGTTTGAGACCTTCAA & \\
R: CATCTCCTGCTCGAAGTCCA & 627 \\
$C Y L D$ & \\
F: TGAGAAAGTTGGCGTTCCCACA & \\
R: GCAGTCTTCGTGCACAGCCTTG & \\
\hline
\end{tabular}

Table 26 pairs of primer sequences were used in mutational analysis of CYLD

\begin{tabular}{ll}
\hline Primer sequences & $\begin{array}{l}\text { Length of } \\
\text { PCR product } \\
(\mathrm{bp})\end{array}$ \\
\hline F1: AGTTCAGGCTTATGGAGCCAAG & 517 \\
R1: GACCACGACCTTCTTCCAGCAA & \\
F2: CGCTTCAGAGGACCCCTGTTA & 785 \\
R2: TTCACAGGAGGAGGCTGGAGTG & \\
F3: GCCTCCCAAACTTGCCTTTAT & 620 \\
R3: AGTGCCTCTGAAGGTTCCATCC & \\
F4: TCGCTGGACTGGAACTGGAAGA & 879 \\
R4: GCCCTCCACATATACCGGCAC & \\
F5: TGAGAAAGTTGGCGTTCCCACA & 627 \\
R5: GCAGTCTTCGTGCACAGCCTTG & \\
F6: AGTCACCCCATGCCCAGAAGT & 582 \\
R6: TTCCTCAAGGCAACCGTTAGC & \\
\hline
\end{tabular}

instructions. cDNA was synthesized by using Transcriptor First Strand cDNA Synthesis Kit (Roche, Mannheim, Germany). Different sets of primer pairs were used for quantitative expression analysis. $\beta$-actin was used as internal control. For the expression analysis of CYLD gene, primer sequences are shown in Table 1 . The thermal profile for CYLD expression initiated with a $10 \mathrm{~min}, 95^{\circ} \mathrm{C}$ denaturation step, followed by 35 cycles of $5 \mathrm{~s}$ at $95{ }^{\circ} \mathrm{C}$ (denaturation); $10 \mathrm{~s}$ at $55^{\circ} \mathrm{C}$ (annealing); and $15 \mathrm{~s}$ at $72{ }^{\circ} \mathrm{C}$ (extension). Each experiment was done in triplicate.

\section{Sequencing of coding region of CYLD}

The CYLD gene encodes 20 exons. Mutational hotspots frequently occur between exons 9-20. The CYLD coding sequences were amplified by PCR using 6 primer pairs covering all exons of the human CYLD gene. Primer sequences are listed in Table 2. The thermal profile for the PCR reactions is as follows: $10 \mathrm{~min}, 95{ }^{\circ} \mathrm{C}$ denaturation step, followed by 40 cycles of $15 \mathrm{~s}$ at $95^{\circ} \mathrm{C}$ for denaturation and $1 \mathrm{~min}$ at $60{ }^{\circ} \mathrm{C}$ for annealing. All PCR samples were run in $1 \%$ agarose gel. PCR products were sequenced by 
bi-directional sequencing (Macrogen, Amsterdam, Netherland). Sequence results were compared with CYLD mRNA reference sequence (NM_015247.2).

\section{Silencing of Dvl genes}

BLOCK-iT ${ }^{\mathrm{TM}}$ RNAi Designer software (Invitrogen) was used to design Dvl1, Dvl2, and Dvl3 siRNA targets approximately 20 bp long. Single strand siRNA oligonucleotides were annealed in a $1 \times$ annealing buffer ( $1 \mathrm{M}$ Tris, $\mathrm{pH} 8.0$, $0.5 \mathrm{M}$ EDTA, $5 \mathrm{M} \mathrm{NaCl}$, DNase/RNase free $\mathrm{dH}_{2} \mathrm{O}$ ). A pool of Dvl siRNA oligonucleotides were transfected into cells by electroporation (Neon Transfection System, Invitrogen). 100 pmol equally mixed siRNA oligonucleotides were added to a homogenized cell pellet of $5 \times 10^{5}$ cell $/ \mathrm{ml}$ density in a micro centrifuge tube. The cell pellet was re-suspended with Resuspension Buffer R. Transfected cell suspensions were added to antibiotic free $10 \%$ FBS containing RPMI1640 cell medium. Cells were incubated for $48 \mathrm{~h}$ before analyses. Input values for the electroporation of each cell line are $1450 \mathrm{~V}, 10 \mathrm{~ms}, 3$ pulses. Scrambled RNA was used as non-target RNAi control. Transfected siRNA sequences are given in Table 3.

\section{Protein extraction and western blotting}

MEG01 cells were grown to $80 \%$ confluency. After $48 \mathrm{~h}$, siRNA treated and untreated cells were washed with ice cold PBS and lysed using PYLB buffer containing $50 \mathrm{mM}$ HEPES (pH 7,6), $50 \mathrm{mM} \mathrm{NaF,} 5 \mathrm{mM}$ EDTA, $10 \mathrm{mM}$ Sodium pyrophosphate (NaPPi), 1\% Triton-X, $50 \mathrm{mM}$ $\mathrm{NaCl}$, protease and phosphatase inhibitors (Applichem GmbH, Germany). Cell lysates were centrifuged at 14,000 rpm for $15 \mathrm{~min}$. Protein concentration was measured by Bradford assay method (Applichem, Darmstadt, Germany). $\beta$-actin was used as a loading control. About $30 \mu \mathrm{g}$ of protein was loaded in to each well of an $10 \%$ SDS-PAGE gel. For lysates of cells overexpressing Dvl proteins, only $20 \mu \mathrm{g}$ of protein was loaded. Gel electrophoresis was run at $100 \mathrm{~V}$ for $1 \mathrm{~h}$. Proteins were transferred to a polyvinylidene difluoride membrane (PVDF) and then blocked with $5 \%$ nonfat dried milk powder at room temperature for $1 \mathrm{~h}$. Membranes were incubated overnight at $4{ }^{\circ} \mathrm{C}$ with $\beta$-actin $(1 / 1000$, Cell Signaling Technologies), CYLD (1/1000, Cell Signaling Technologies), Dvl1, Dvl2 and Dvl3 (1/1000, Santa Cruz) antibodies. Goat antirabbit and goat anti-mouse secondary antibodies were used against first antibody (Cell Signaling Technologies, 1/10,000). Prestained Protein Ladder was used (Thermo Fisher Scientific \#26619c). Protein bands were detected by Luminata Forte Western HRP Substrate (Millipore, Billerica, MA). Densitometric analysis of detected protein bands were performed by ImageJ software (http://imagej. nih.gov/ij/).

\section{Overexpression of Dvl2 and Dvl3 genes}

Dvl2 and Dvl3 genes were cloned into the eukaryotic expression vector $\mathrm{pEGFP-C1}$ and transfected into MEG01 cells by electroporation. $70-80 \%$ confluent cells were seeded in a 6 well plate. Plating medium was $2 \mathrm{ml}$ and the cells were cultured in an antibiotic free RPMI1640. pEGFP-C1-Dvl2/ pEGFP-C1-Dvl3 vectors were equally mixed (1:1) and transiently transfected by electroporation. $0,5 \mu \mathrm{g} / \mathrm{ml}$ empty pEGFP-C 1 vector was also transfected as control. The same electrical force mentioned previously was applied to the cells. After electroporation cells were incubated for $48 \mathrm{~h}$ before analyses. Transfection efficiency was determined by DIC microscope (Olympus) and Western Blot.

\section{Immunofluorescence analysis}

Cells were fixed with $4 \%$ paraformaldehyde for $15 \mathrm{~min}$ and permeabilized in $0.1 \%$ Triton-x for 15 min on ice. Blocking was carried out in $1 \% \mathrm{BSA}$ for $1 \mathrm{~h}$ at room temperature. Cells were incubated with Dvl2 (Santa Cruz, 1/1000) and $\beta$ catenin (BD, 1/1000) primer antibodies. Green fluorescent dye conjugated secondary antibodies were added to label the indicated proteins (Invitrogen, Alexa Fluor488 goat anti mouse and rabbit). Nuclei were counterstained with DAPI (Applichem).
Table 3 siRNA and nontargeting siRNA sequences used in Dvls silencing

\begin{tabular}{lllc}
\hline Gene name (RefSeq) & siRNA sequence & Binding sequence & Exon \\
\hline Dvl1 (NM_004421) & 5'-AAGAUUAUCUACCACAUtt-3' & $58-78$ & 1 \\
& 3'-ggUUCUAAUAGAUGGUGUA-5' & & \\
Dvl2 (NM_004422) & 5'-GUCAACAAGAUCACCUUtt-3' & $1754-1774$ & 13 \\
& 3'-ggCAGUUGUUCUAGUGGAA-5' & & \\
Dvl3 (NM_004423) & 5'-GUCAACAAGAUCACCUUtt-3' & $1579-1599$ & 13 \\
& 3'-ggCAGUUGUUCUAGUGGAA-5' & & \\
ScrambledsiRNA & 5'-GACAAUAGACACCUCUUtt-3' & - & - \\
& 3'-ggCUGUUAUCUGUGGAGAA-5' & & \\
\hline
\end{tabular}




\section{Results}

\section{CYLD mRNA is expressed in CML cell lines}

Previous studies have shown that activation of the Wnt signalling pathway plays a key role in disease progression to blastic phase. In aim to investigate the possibility of $\beta$-catenin activation in blastic phase CML due to deregulation of Dvl proteins through CYLD; we first determined whether CYLD was expressed in blastic phase CML cells. K562, MEG01 and KU812 cells were analyzed by RT-PCR for CYLD mRNA expression. All three blastic phase CML lines were shown to express the CYLD gene (Fig. 1).

\section{Mutational analysis of CYLD coding region in CML cell lines}

Total RNA was extracted and converted to cDNA from K562, MEG01 and KU812 cell lines and PCR was performed using the specific primers given in Table 2 . We used

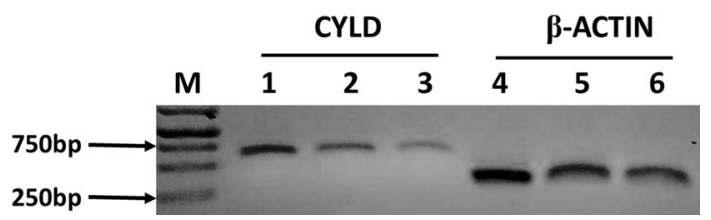

Fig. 1 K562, MEG01and KU812 cell lines express CYLD mRNA. CYLD mRNA expression was assessed in three different CML cell lines. PCR results showing CYLD ( 1 K562, 2 MEG01, 3 Ku812) and $\beta$-actin (4 K562, 5 MEG01, 6 KU812) gene expression on $1 \%$ agarose gel. $\beta$-actin was used as loading control (bands; CYLD: $627 \mathrm{bp}$, $\beta$-actin: 318 bp; M-Marker: O' Range Ruler 1 kb DNA ladderThermo Scientific)

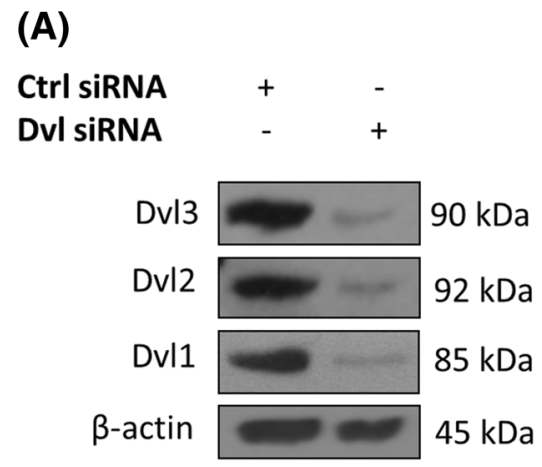

Fig. 2 Silencing Dvl proteins decreases CYLD expression as shown by Western blot. a A significant decrease in Dvl genes were observed after Dvls silencing in MEG01 cell line. b CYLD protein was shown by Western blot in MEG01 cells and control scrambled RNAi vector transfected cell lines (CYLD primer antibody, 1/1000, Cell Signaling
6 primer pairs to cover the coding sequences of the CYLD gene. Primers were designed from exon-exon junction regions to eliminate false positive results caused by genomic DNA contamination. PCR products were sequenced bidirectionally. We determined that none of the cell lines carry mutations in the coding region of the CYLD gene.

\section{Effect of silencing Dvl1, -2, -3 proteins on $\beta$-catenin subcellular localization and CYLD protein levels}

Dvl1, $-2,-3$ genes were silenced by siRNA oligonucleotides in MEG01 cells. Cells were collected after $48 \mathrm{~h}$ following transfection and protein expression was analyzed. Dvl genes were shown to be downregulated successfully in MEG01 cells (Fig. 2a). Interestingly a 53\% reduction was observed in CYLD protein expression at the 48th hour in Dvl siRNA transfected cells when compared to scrambled siRNA transfected controls (Fig. 2b). The effect of silencing Dvl proteins on $\beta$-catenin was investigated by immunofluorescent microscopy. Neither an increase in $\beta$-catenin protein levels; nor a change in subcellular localization was observed in cells transfected with Dvl pool siRNA (Fig. 3), confirming that the $\mathrm{Wnt} / \beta$-catenin canonical pathway has not been activated by the procedure. While previous reports have described CYLD as a regulator of DVL proteins; our data suggests the presence of a more complicated reciprocal regulatory mechanism.

\section{Effect of Dvl2 and Dvl3 overexpression on CYLD protein level}

Dvl2 and Dvl3 genes were cloned into a pEGFP-C1 vector and overexpressed after being transfected into CML cell

(B) CYLD downregulation

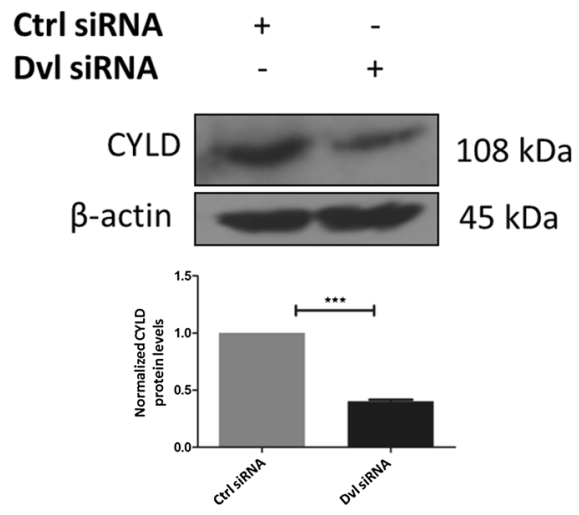

Technologies). A decrease in CYLD protein level was observed after Dvl1, -2, -3 genes silencing when compared with control. Primary antibodies used: $\beta$-actin $(1 / 1000$, CST), Dvl1, 2,3 (1/1000, Santa Cruz) 

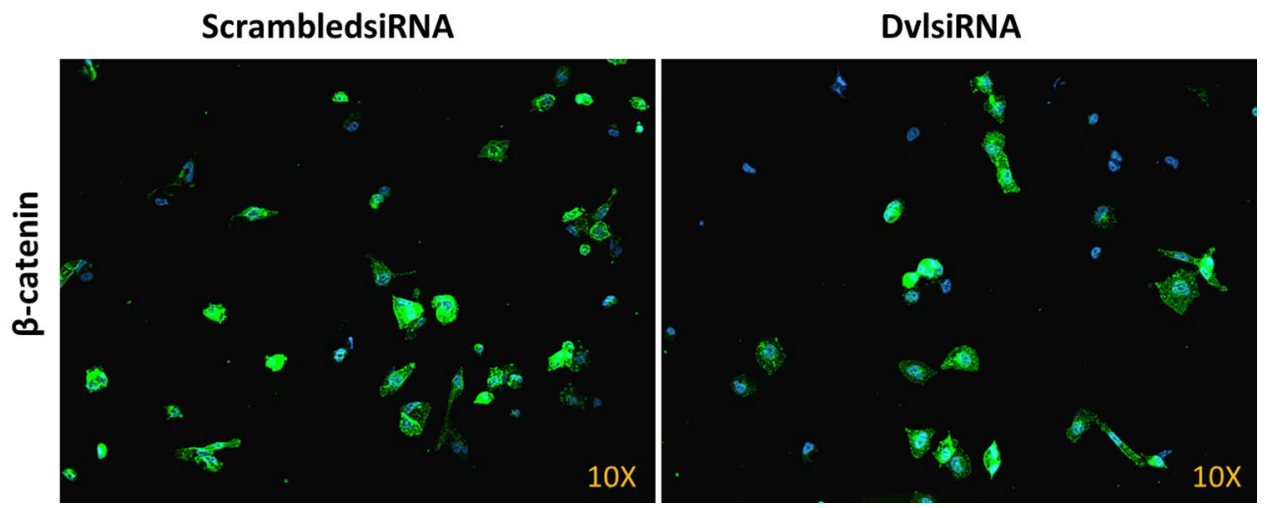

Fig. 3 Immunofluorescence microscopy images of $\beta$-catenin localization before and after silencing Dvl proteins. Following silencing of Dvl proteins in MEG01 cells, cytoplasmic localization of and expression level of $\beta$-catenin is not altered; showing that the $\mathrm{Wnt} / \beta$-catenin canonical pathway has not been activated following siRNA transfection. Nucleus stained with DAPI; ( $\beta$-catenin antibody, BD Transduction Laboratories Cat No: 610153)

self-renewal and homeostasis [1]. Pathway activation leads to $\beta$-catenin being stabilized and its accumulation in the cytoplasm and translocation to the nucleus where it regulates the activity of several transcription factors. Following the binding of specific Wnt ligands with their partner Frizzled $(\mathrm{Fz})$ receptors, one of the first steps of the signaling pathway is Fz receptor-mediated plasma membrane recruitment and activation of the cytoplasmic effector Dishevelled (Dvl). Dvl proteins play central role in relaying Wnt signals from the plasma membrane to different cytoplasmic effectors [18]. In mammals there are three members of Dvl family: Dvl1, -2 and -3 [19]. They are activated by phosphorylation in response to Wnt signaling; whereas the ubiquitination of Dvl proteins leads to an effective inhibition of the $\beta$-catenin destruction complex [20]. Dvl ubiquitination can be antagonized by various de-ubiquitinases including the CYLD tumor suppressor protein, resulting in inhibited Wnt signaling. CYLD, has

$\beta$-catenin/Wnt signaling pathway is crucial for embryogenesis, development, cell proliferation, differentiation,

Fig. 4 CYLD expression increases with Dvl2 and Dvl3 overexpression. a Dvl2 and $\mathrm{Dvl} 3$ genes cloned in to $\mathrm{pEGFP}$ vector are overexpressed following transfection The lower band observed at the same height when compared to the control represent the cellular Dvl2 and Dvl3 proteins; while the upper band with a higher molecular weight corresponds to the transfected Dvl proteins containing the GFP tag. b Protein expression of CYLD is upregulated with Dvl2 and Dvl3 over-expression
(A)

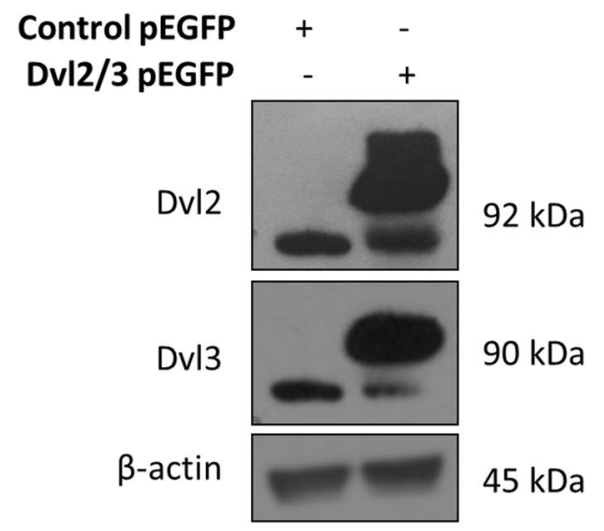

(B) CYLD upregulation
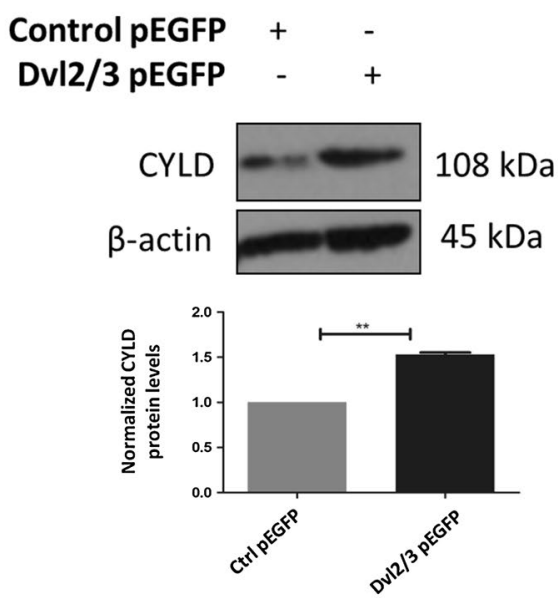
been defined as a tumor suppressor gene, and a negative regulator of in $\mathrm{Wnt} /$ beta-catenin signaling that regulates Dvl activity [11].

CYLD was originally reported as a tumor suppressor gene [9] that is mutated in familial cylindromatosis, a rare autosomal dominant disorder associated with numerous benign skin tumors. It was shown to regulate cell proliferation and survival. CYLD is known to interact with several proteins such as TNF-receptor-associated factor proteins and NEMO protein [14]. The CYLD gene product is a deubiquitinating enzyme that has specificity for Lys63 chains. CYLD is now known to regulate various signaling pathways such as Wnt/ $\beta$-catenin signaling, NF- $\kappa B$ signaling, TGF- $\beta$ signaling, Bcl2 signaling and the MAPK pathway, by deubiquitinating upstream regulatory factors. Downregulation of CYLD has been reported in several types of cancer including lung [21], breast [22], skin cancers [23], colon cancer, hepatocellular carcinomas [24] and multiple myeloma [25]. A close relation between CYLD and Dvl-linked Wnt signaling was defined by Taurello et al. [11]. They reported that CYLD depletion leads to hyper ubiquitination of Dvl proteins and Wnt pathway activation, resulting in $\beta$-catenin overexpression which in turn could lead to tumorigenesis and/or progression of an existing malignancy. However, the role and significance of CYLD expression in myeloid malignancies, including chronic myeloid leukemia (CML) has not been investigated and is thus unknown.

CML is a triphasic myeloproliferative disorder clinically characterized by an initial chronic phase which progresses to an accelerated phase and terminally blast crisis [26]. The hallmark of the disease is the $\mathrm{t}(9 ; 22)$ (q34;q11) translocation [27] which results in the formation of a BCR/ABL fusion gene [28]. The Bcr/Abl oncoprotein is a constitutively active tyrosine kinase causing the unregulated activation of several signaling pathways [29]. CML was the first hematological malignancy in which aberrant $\mathrm{Wnt} / \beta$-catenin signaling was first defined [15], and in which $\beta$-catenin over-expression was shown to be responsible for blastic transformation, disease progression and resistance to therapy [17].

In the present study we aimed to investigate the role CYLD had -if any- in Dvl mediated $\beta$-catenin signaling in CML cell lines K562, MEG01 and KU821. All three cell lines are obtained from patients in blastic crisis. We first evaluated CYLD mRNA expression and showed that all three cell lines express CYLD. To exclude the possibility of any mutations in the CYLD coding sequence, mutational analyses were performed and we showed that the CYLD gene did not carry any mutations in its coding sequences in K562, MEG01 and KU821 cell lines. Our data shows that CYLD is expressed in CML cell lines and its coding sequence is not mutated; indicating that the CYLD gene may not play a role in unregulated Wnt signaling observed in CML. In the literature CYLD mutation/ aberrations has been associated with different types of cancer; on the contrary we couldn't observe any aberrations in CML cell lines.

Dvl-CYLD protein interactions were reported by several studies, defining CYLD as a regulator of Dvl proteins through de-ubiquitination. We silenced Dvl genes with siRNA oligonucleotides in MEG01 cells. Interestingly CYLD mRNA and protein expression was also shown to be downregulated over twofolds in Dvl siRNA transfected cells when compared to scrambled siRNA transfected controls. While previous reports have described CYLD as a regulator of Dvl proteins; our data suggests the presence of a more complicated reciprocal regulatory mechanism. In an opposite approach we overexpressed Dvl genes and observed that CYLD protein expression also increased. The positive correlation between the expression patterns of CYLD and Dvl protein supports the presence of a complex modulatory mechanism in which the expression of both genes have a positive regulatory effect on each other. Although previous reports have shown Dvl regulation by CYLD, this is the first study to suggest that the observed regulatory function may be reciprocal. Our data reveals another layer of complexity in the function of Dvl proteins in which further research will be required to understand its interactions with CYLD.

Acknowledgements This study was financially supported by The Scientific and Technological Research Council of Turkey (TUBITAK) (Project Code: 1001, Grant Number: 114Z225).

\section{Compliance with ethical standards}

Conflict of interest The authors declare that they have no conflict of interest.

\section{References}

1. Logan CY, Nusse R (2004) The Wnt signaling pathway in development and disease. Annu Rev Cell Dev Biol 20:781-810

2. Gao C, Chen Y-G (2010) Dishevelled: the hub of Wnt signaling. Cell Signal 22(5):717-727

3. Wharton KA (2003) Runnin'with the Dvl: proteins that associate with Dsh/Dvl and their significance to Wnt signal transduction. Dev Biol 253(1):1-17

4. Komander D (2010) CYLD tidies up dishevelled signaling. Mol Cell 37(5):589-590

5. Khan AS, Hojjat-Farsangi M, Daneshmanesh AH, Hansson L, Kokhaei P, Österborg A, Mellstedt H, Moshfegh A (2016) Dishevelled proteins are significantly upregulated in chronic lymphocytic leukaemia. Tumor Biol 39(7):11947-11957

6. Li J, Guo G, Li J, Hao J, Zhang J, Guo Y, Yu H (2014) The expression and significance of dishevelled in human glioma. J Surg Res 192(2):509-514

7. Uematsu K, He B, You L, Xu Z, McCormick F, Jablons DM (2003) Activation of the Wnt pathway in non small cell lung cancer: evidence of dishevelled overexpression. Oncogene 22(46):7218-7221 
8. Zhou G, Ye J, Sun L, Zhang Z, Feng J (2016) Overexpression of Dishevelled-2 contributes to proliferation and migration of human esophageal squamous cell carcinoma. J Mol Histol 47(3):287-295

9. Bignell GR, Warren W, Seal S, Takahashi M, Rapley E, Barfoot R, Green H, Brown C, Biggs PJ, Lakhani SR (2000) Identification of the familial cylindromatosis tumour-suppressor gene. Nat Genet 25(2):160-165

10. Kovalenko A, Chable-Bessia C, Cantarella G, Israël A, Wallach D, Courtois G (2003) The tumour suppressor CYLD negatively regulates $\mathrm{NF}-\kappa \mathrm{B}$ signalling by deubiquitination. Nature 424(6950):801-805

11. Tauriello DV, Haegebarth A, Kuper I, Edelmann MJ, Henraat M, Canninga-van Dijk MR, Kessler BM, Clevers H, Maurice MM (2010) Loss of the tumor suppressor CYLD enhances Wnt/ $\beta$ catenin signaling through K63-linked ubiquitination of Dvl. Mol Cell 37(5):607-619

12. Massoumi R (2011) CYLD: a deubiquitination enzyme with multiple roles in cancer. Future Oncol 7(2):285-297

13. Sun S (2010) CYLD: a tumor suppressor deubiquitinase regulating NF- $\mathrm{KB}$ activation and diverse biological processes. Cell Death Differ 17(1):25-34

14. Nagy N, Farkas K, Kemény L, Széll M (2015) Phenotype-genotype correlations for clinical variants caused by CYLD mutations. Eur J Med Genet 58(5):271-278

15. Jamieson CH, Ailles LE, Dylla SJ, Muijtjens M, Jones C, Zehnder JL, Gotlib J, Li K, Manz MG, Keating A (2004) Granulocytemacrophage progenitors as candidate leukemic stem cells in blastcrisis CML. N Engl J Med 351(7):657-667

16. von Bubnoff N, Duyster J (2010) Chronic myelogenous leukemia. Dtsch Arztebl Int 107:114-121

17. Ashihara E, Takada T, Maekawa T (2015) Targeting the canonical $\mathrm{Wnt} / \beta$-catenin pathway in hematological malignancies. Cancer Sci 106(6):665-671

18. Fukumoto S, Hsieh C-M, Maemura K, Layne MD, Yet S-F, Lee K-H, Matsui T, Rosenzweig A, Taylor WG, Rubin JS (2001) Akt participation in the Wnt signaling pathway through Dishevelled. J Biol Chem 276(20):17479-17483

19. Kishida S, Yamamoto H, Hino S-i, Ikeda S, Kishida M, Kikuchi A (1999) DIX domains of Dvl and Axin are necessary for protein interactions and their ability to regulate $\beta$-catenin stability. Mol Cell Biol 19(6):4414-4422

20. Gao C, Xiao G, Hu J (2014) Regulation of Wnt/ $\beta$-catenin signaling by posttranslational modifications. Cell Biosci 4(1):13

21. Zhong S, Fields C, Su N, Pan Y, Robertson KD (2007) Pharmacologic inhibition of epigenetic modifications, coupled with gene expression profiling, reveals novel targets of aberrant DNA methylation and histone deacetylation in lung cancer. Oncogene 26(18):2621-2634

22. Hayashi M, Jono H, Shinriki S, Nakamura T, Guo J, Sueta A, Tomiguchi M, Fujiwara S, Yamamoto-Ibusuki M, Murakami K-i (2014) Clinical significance of CYLD downregulation in breast cancer. Breast Cancer Res Treat 143(3):447-457

23. Alameda J, Fernandez-Acenero M, Moreno-Maldonado R, Navarro M, Quintana R, Page A, Ramírez A, Bravo A, Casanova M (2011) CYLD regulates keratinocyte differentiation and skin cancer progression in humans. Cell Death Dis 2(9):e208

24. Hellerbrand C, Bumes E, Bataille F, Dietmaier W, Massoumi R, Bosserhoff AK (2006) Reduced expression of CYLD in human colon and hepatocellular carcinomas. Carcinogenesis 28(1):21-27

25. Jenner MW, Leone PE, Walker BA, Ross FM, Johnson DC, Gonzalez D, Chiecchio L, Cabanas ED, Dagrada GP, Nightingale M (2007) Gene mapping and expression analysis of $16 \mathrm{q}$ loss of heterozygosity identifies WWOX and CYLD as being important in determining clinical outcome in multiple myeloma. Blood 110(9):3291-3300

26. Schiffer C, Hehlmann R, Larson R (2003) Perspectives on the treatment of chronic phase and advanced phase CML and Philadelphia chromosome positive ALL1. Leukemia 17(4):691-699

27. Rowley JD (1973) A new consistent chromosomal abnormality in chronic myelogenous leukaemia identified by quinacrine fluorescence and Giemsa staining. Nature 243(5405):290-293

28. Lugo TG, Pendergast A-M, Muller AJ, Witte ON (1990) Tyrosine kinase activity and transformation potency of bcr-abl oncogene products. Science 247(4946):1079-1082

29. Chang G, Zhang H, Wang J, Zhang Y, Xu H, Wang C, Zhang H, Ma L, Li Q, Pang T (2013) CD44 targets Wnt/ $\beta$-catenin pathway to mediate the proliferation of K562 cells. Cancer Cell Int 13(1): 117 